\title{
EL TRABAJO FIN DE GRADO (TFG) EN LA FORMACIÓN DEL MAESTRO. LA ACCIÓN TUTORIAL COMO PIEZA CLAVE DEL PROCESO ${ }^{1}$
}

\section{FINAL DEGREE PROJECT IN TEACHER TRAINING. TUTORIAL ACTION AS KEY PART OF PROCESS}

\author{
Magdalena Sáenz-de-Jubera-Ocón ${ }^{2}$ \\ Rosa Ana Alonso- Ruiz ${ }^{3}$ \\ María Ángeles Valdemoros-San-Emeterio ${ }^{4}$ \\ Eva Sanz-Arazuri ${ }^{5}$ \\ Ana Ponce-de-León-Elizondo ${ }^{6}$ \\ Universidad de La Rioja (España)
}

\section{Resumen}

\begin{abstract}
El Trabajo Fin de Grado (TFG) supone para el alumnado universitario español una innovación educativa en la que la formación en tareas de diseño, planificación y ejecución es un aspecto fundamental para considerar por parte de los tutores. El objetivo del artículo es presentar una experiencia de formación teórico-práctica que se está desarrollando en los grados de educación infantil y primaria de la Universidad de La Rioja (España), así como mostrar los resultados obtenidos hasta el momento utilizando los TFG como una oportunidad para gestar proyectos de ocio saludable en familia. Se plantea un modelo de tutoría basado en sesiones individuales y grupales organizando actividades formativas en formato talleres-seminarios con los docentes y el alumnado implicado. En estas sesiones se promueven iniciativas colectivas que se concretan en numerosas propuestas didácticas adaptadas a las necesidades de niños y jóvenes en torno a cuatro grandes ejes: ocio cultural, juegos tradicionales, actividad física y lectura compartida. A grandes rasgos, se concluye que el modelo planteado es un recurso educativo de gran valor que permite trabajar de forma eficiente en la realización de los TFG, y que las propuestas didácticas planteadas obtienen interesantes ventajas educativas, sociales y culturales que las hacen transferibles a otros contextos.
\end{abstract}

Palabras clave: formación, docente, tutoría, ocio, familia

1 El texto que presentamos se vincula al proyecto de investigación De los tiempos educativos a los tiempos sociales: la construcción cotidiana de la condición juvenil en una sociedad de redes. Problemáticas especificas y alternativas pedagógico-sociales (proyecto coordinado EDU201239080-C07-00) y al subproyecto De los tiempos educativos a los tiempos sociales: la cotidianidad familiar en la construcción del ocio físicodeportivo juvenil (EDU2012-39080-C07-05), cofinanciado en el marco del Plan Nacional I+D+i, con cargo a una ayuda del Ministerio de Economía y Competitividad, y por el Fondo Europeo de Desarrollo Regional (FEDER, 2007-2013).

2 Doctora en ciencias de la educación, Universidad de La Rioja. Correo electrónico: m-magdalena.saenz-de-jubera@unirioja.es.

3 Doctora en ciencias de la educación, Universidad de La Rioja. Correo electrónico: rosa-ana.alonso@unirioja.es.

4 Doctora en ciencias de la educación, Universidad de La Rioja. Correo electrónico: maria-de-los-angeles.valdemoros@unirioja.es.

5 Doctora en ciencias de la actividad física y deporte, Universidad de La Rioja. Correo electrónico: eva.sanz@unirioja.es.

6 Doctora en filosofía y ciencias de la educación, Universidad de La Rioja. Correo electrónico: ana.ponce@unirioja.es. 


\section{Abstract}

The Trabajo Final de Grado (TFG; in English, final degree project) supposes for the Spanish university students an educational innovation where the training for design, planning and implementation tasks is a key aspect to consider by tutors. The aim of the paper is to present an experience of theoretical and practical training which is currently being developed in the primary grades at University of La Rioja (Spain) children's education, besides showing the results obtained up to now using this framework as an opportunity to bring forward healthy family entertainment projects. A mentoring model based on individual and group sessions is proposed by organizing training activities in workshops-seminars format with teachers and students involved. In these sessions collective initiatives that are specified in numerous educational proposals adapted to the needs of children and youth around four major axes are promoted: cultural leisure, traditional games, physical activity and shared reading. Broadly speaking, it is concluded that the proposed model is an educational resource of great value that can work efficiently in conducting the TFG, and that the educational proposals raised obtain interesting educational, social and cultural advantages that make them transferable to other contexts.

Keywords: training, teacher, mentoring, leisure, family

Fecha de recepción: 2 de diciembre de 2015

Fecha de aprobación: 6 de mayo de 2016

Para citar este artículo:

Sáenz-de-Jubera-Ocón, M., Alonso- Ruiz, R.A., Valdemoros-San-Emeterio, M.A., Sanz-Arazuri, E. \& Ponce-de-León, A. (2016).

El trabajo fin de grado en la formación del maestro. La acción tutorial como pieza clave del proceso. Lúdica Pedagógica, $24,47-55$.

\section{INTRODUCCIÓN}

El establecimiento del Espacio Europeo de Educación Superior ha producido grandes cambios en el sistema universitario español. Uno de los más importantes es la introducción de la asignatura Trabajo Fin de Grado (TFG) en los planes de estudio de todas las titulaciones. Ello supone un novedoso escenario que precisa de modelos que orienten al profesorado y al alumnado universitario en su proceso de elaboración.

La normativa (Real Decreto 861/2010) consagra que el TFG tendrá entre 6 y 30 créditos, que ha de realizarse durante la fase final del plan de estudios y ha de estar orientado a la evaluación de competencias asociadas al grado en cuestión. Las disposiciones en lo relativo a la elaboración y la evaluación son reguladas por cada universidad, como consecuencia del ejercicio de su autonomía.

En el contexto descrito, cada universidad establece una serie de normas de carácter general sobre la elaboración y la defensa del TFG, con el fin de unificar los criterios y los procedimientos que garanticen una actuación homogénea en la planificación y la evaluación de los TFG.
Posteriormente, cada Junta de Facultad desarrolla esta normativa relativa a la definición, la elaboración, la defensa, la calificación y la tramitación administrativa de los trabajos, con el fin de adecuarla a las características propias de cada uno de los títulos de grado.

Si bien la asignatura contempla la realización de modo individual de proyectos, de trabajos experimentales, de revisión e investigación bibliográfica, nuestra propuesta se focaliza en el diseño, la elaboración, la implementación y la evaluación de proyectos educativos, por considerar que de ese modo el TFG se establece dentro del marco idóneo para que el alumnado reconstruya los contenidos, las capacidades, las competencias pedagógicas y las habilidades adquiridas durante el grado, y se sitúe en contextos próximos a un entorno laboral propio de la sociedad de la información y la comunicación; asimismo, se promueven la responsabilidad, el autoconocimiento, la creatividad, la toma de decisiones y la valoración de riesgos, así como la decisión de hacer frente a los posibles problemas (Ponce de León, Sanz \& Valdemoros, 2013). En definitiva, se presenta como un espacio y un tiempo idóneos para que el estudiante, 
acompañado por uno o más tutores-profesores de la propia universidad, o externos a ella, demuestre su capacitación como profesional finalizando su proceso inicial de formación (Rekalde, 2011).

En el caso que nos ocupa, los TFG desarrollan propuestas didácticas de ocio saludable en familia, adaptadas a las necesidades de los niños y jóvenes, de modo que ofrezcan oportunidades pedagógicas que optimicen tanto el desarrollo personal como las interacciones familiares. Dichas propuestas giran en torno a cuatro grandes ejes: ocio cultural, juegos tradicionales, actividad física y lectura compartida.

El ocio es un derecho fundamental ligado al crecimiento personal y social (Álvarez, Fernández-Villarán \& Mendoza, 2014; Caride, 2012; Carrera, 2009; Clerton de Oliveira, Francileudo \& Ibiapina, 2014; Cuenca, 2000, 2009, 2014; Otero, 2009; World Leisure and Recreation Association, 1994) que se vincula a valores como la satisfacción, la libertad y la voluntariedad (Cuenca \& Goytia, 2012), por lo cual se hace trascendente para el desarrollo humano en lo individual, lo colectivo, lo cognitivo y lo emocional (Caride, 1998; Cuenca, 1997, 2009; Quintana, 1991). Se constituye, por tanto, en una experiencia humana con un fin en sí misma, elegida libremente y con potencial para generar satisfacción personal y disfrute reflexivo.

La institución familiar es agente educativo por excelencia y posee una gran capacidad pedagógica (Álvarez \& Rodríguez, 2008), lo que brinda un ambiente idóneo para la cimentación del ocio como experiencia valiosa de desarrollo humano de los hijos desde la infancia hasta la juventud (Cuenca, 2013; Valdemoros, Ponce de León, Sanz \& Caride, 2014).

Las actividades de ocio compartido en familia reportan importantes beneficios, como la comunicación y la mejora de las interacciones familiares (Zabriskie \& McCormick, 2001), el progreso de habilidades de resolución de problemas o conflictos (Mactavish \& Schleien, 1998), una mejor cohesión, una mejor adaptabilidad y un mejor funcionamiento familiar (Agate, Zabriskie \& Eggett, 2007; Zabriskie \& McCormick, 2001) o una mayor satisfacción con la vida familiar (Agate, Zabriskie, Agate \& Poff, 2009; Zabriskie \& McCormick, 2003).

El TFG supone para el alumnado universitario una innovación educativa de gran calado en la que la formación en tareas de diseño, planificación y ejecución es un aspecto fundamental para considerar por parte de los tutores, a lo cual hay que añadir la carga de trabajo que supone para ellos llevar a cabo la tutorización de varios TFG a la vez durante un periodo corto, así como evaluar - de forma compartida con el tribunal- las competencias, las capacidades y las habilidades antes expuestas.

En tal escenario, se concibe el desarrollo del TFG como un proceso de aprendizaje donde se integran competencias específicas y, fundamentalmente, transversales, con especial énfasis en la tutorización del alumnado, asumiendo la tutoría como "un espacio educativo de reflexión donde el alumnado puede exponer sus percepciones, reflexiones, problemas y objetivos, y el tutor/a puede enseñarle a aprender a aprender" (Rekalde, 2011, p. 189).

En el contexto descrito, el objetivo de este trabajo es presentar una experiencia de formación teórico-práctica que se está desarrollando en los grados de educación infantil y primaria de la Universidad de La Rioja, para trabajar de forma más eficiente en la realización de los TFG en educación, así como mostrar los resultados obtenidos hasta el momento utilizando este marco como una oportunidad para gestar proyectos de ocio saludable en familia.

\section{PROCESO METODOLÓGICO}

Este trabajo se ubica en el proyecto de innovación docente de la Universidad de La Rioja El aprendizaje del maestro mediante proyectos de ocio saludable familiar. En dicho proyecto se cuenta con profesorado tanto de esta universidad como externos a ella; también, con personal de instituciones oficiales relacionadas con la educación y la salud, buscando crear redes de soporte colaborativo entre el profesorado y agentes sociales activos. Asimismo, los miembros de este proyecto conciertan varias plazas con el alumnado en el tema definido dentro del proyecto. En total, han sido 20 plazas comprometidas con los estudiantes, relacionadas con el ocio familiar saludable.

A partir de las necesidades y las demandas de los estudiantes, se diseña una acción tutorial formativa del proceso de elaboración del TFG articulando este espacio educativo de manera individual y en grupo, y abordando ambas modalidades en diferentes momentos del proceso. 
En tutoría grupal se organizan actividades formativas en formato talleres-seminarios, con todos los docentes de la Universidad de la Rioja, con los profesores externos a través de videoconferencia y con los alumnos tutelados, en los cuales se forma al alumnado en el qué, el por qué, el cómo, el con quién y el para qué del TFG. Los diferentes encuentros se corresponden con la fase inicial (en la que el alumno ha de profundizar sobre el tema por tratar, hacer la búsqueda bibliográfica, plantear los objetivos y relacionarlos con la experiencia adquirida, y abordar el planteamiento y el esquema del trabajo) y con la de progreso (en la que el estudiante va a desarrollar el trabajo siguiendo la fase inicial), en las que se basa el modelo de tutorización de la Universidad de La Rioja, y de las que el tutor lleva a cabo una evaluación de forma continuada (Flores, Martínez-Berbel, Martínez, Pascual \& Sanz, 2013).

El primer seminario que se celebra tiene carácter informativo, con el objeto de cohesionar al grupo, así como para aportar y obtener información de partida: se expone la normativa del TFG; se clarifica el tema objeto de estudio, para que el alumnado elija lo que se adapte a sus intereses; se muestran experiencias que les ayuden a configurar sus propuestas, y se analizan los principales documentos bibliográficos que tratan el tema objeto de estudio, dónde localizarlos, cómo acceder a ellos y cómo gestionar la documentación localizada. Al mismo tiempo que se da respuesta a los interrogantes surgidos en los primeros momentos del proceso, se empieza a definir de una forma más clara el objeto de estudio. Todo ello se hace dentro del marco de un equipo de trabajo integrado por tutores, alumnos y colaboradores externos, para así dar respuesta a una de las competencias transversales por adquirir en la titulación: la relacionada con la capacidad para trabajar en equipo, integrarse en grupos interdisciplinares y colaborar con profesionales de otros ámbitos de conocimiento.

En el segundo seminario-taller, desde el punto de vista de los tutores, se hace hincapié tanto en el contenido del TFG (qué debe expresar la introducción, cómo redactar los objetivos y cómo tomar decisiones metodológicas - técnicas más apropiadas-, cómo atender a la forma - redacción, uso del lenguaje y calidad de la presentación-). Por su parte, los alumnos exponen su planteamiento sobre el objeto de estudio y las principales conclusiones obtenidas tras la búsqueda de experiencias similares realizadas en distintas comunidades autónomas por entidades públicas y privadas; así se pone de manifiesto que la oferta de actividades de ocio en familia no se ajusta, en muchos casos, a la edad ni a los intereses de los destinatarios (niños y jóvenes). Se toman decisiones para crear propuestas de intervención didáctica que partan desde el centro educativo en colaboración con la familia. Se organizan los grupos de trabajo por iniciativas afines, constituidos por cuatro o cinco alumnos y un docente, con el fin de compartir recursos y propiciar la gestación de iniciativas colectivas que favorezcan sinergias que optimicen el proceso y los resultados, y así responder a otra de las competencias transversales, relacionada con la capacidad para la comunicación interpersonal y la conciencia de las capacidades, así como de los recursos propios y de los otros.

La tercera sesión grupal se centra en la configuración del diseño de las propuestas de intervención, tratando de convertir las experiencias de ocio en familia en verdaderas oportunidades pedagógicas. Se pretende que la intervención pedagógica y didáctica se adapte a niños y jóvenes, así como a sus conocimientos previos, de modo que suponga un aprendizaje capaz de despertar y mantener el interés por las prácticas de ocio mediante un proceso lúdico. El estudiante adquiere, de esta manera, las capacidades de aplicar los conocimientos a la práctica, diseñar y gestionar proyectos y adaptarse a nuevas situaciones.

En estos talleres-seminarios se facilitan, en un ambiente de confianza y seguridad, la relación próxima, el respeto, el compromiso colectivo y el trabajo cooperativo. Soria (2013) hace una serie de recomendaciones a la hora de abordar actividades formativas del TFG, e insiste en que "La Zona de Desarrollo Próximo y el trabajo entre iguales facilita el aprendizaje y da un soporte inestimable. En las actividades formativas propiciemos procesos cooperativos y no solitarios" (p. 9).

Al finalizar cada una de las tutorías grupales, considerando el peso que tiene el trabajo autónomo en los TFG, se proporciona un acompañamiento a los alumnos, mediante tutorías individualizadas, para solventar situaciones particulares, y es cuando cada estudiante entrega al tutor las "evidencias" correspondientes a lo que se les pide en cada uno de los seminarios, y se comprueba así la adquisición de competencias asociadas a la capacidad para el aprendizaje autónomo, la iniciativa personal, la automotivación y la perseverancia. 
A partir de ahí, las sesiones grupales dan paso a las tutorías individualizadas centradas en la orientación y el asesoramiento, por parte de los tutores, del desarrollo de cada TFG en concreto, para que el alumno, con los documentos informativos previos, así como con la descripción y los análisis realizados, esté preparado para la redacción del informe final. Coincide ello, aproximadamente, con la mitad de la fase de progreso y con la fase final, cuando se entrega la versión definitiva del TFG, para efectuar su defensa.

La tabla 1 sintetiza los aspectos fundamentales de cada una de las fases detalladas en este epígrafe.

Tabla 1. Modelo de tutoría basado en sesiones grupales e individuales.

\begin{tabular}{|c|c|c|c|c|c|c|}
\hline Fase & Semana & Tipo & Tema por tratar & Responsable & $\begin{array}{c}\text { Trabajo para el } \\
\text { estudiante }\end{array}$ & Evidencia escrita \\
\hline$\stackrel{\frac{\pi}{\pi}}{\Xi}$ & 1 & 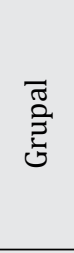 & $\begin{array}{l}\text { Clarificación de dudas respecto a } \\
\text { normativa. } \\
\text { Presentación del tema objeto de } \\
\text { estudio y experiencias similares. } \\
\text { Fuentes de información } \\
\text { bibliográfica. }\end{array}$ & 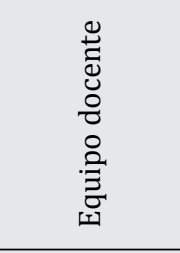 & $\begin{array}{l}\text { Análisis de vivencias, } \\
\text { experiencias y reflexio- } \\
\text { nes anteriores. } \\
\text { Búsqueda de bibliografía } \\
\text { y primeras lecturas. }\end{array}$ & $\begin{array}{l}\text { Concreción de título, tema y } \\
\text { objetivos. }\end{array}$ \\
\hline \multirow{3}{*}{$\frac{\pi}{\mathscr{U}}$} & \multirow{3}{*}{5} & \multirow{3}{*}{ 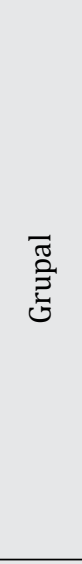 } & $\begin{array}{l}\text { Detalle de estructura y aspectos } \\
\text { formales del TFG (entrega docu- } \\
\text { mentación elaborada al efecto). }\end{array}$ & 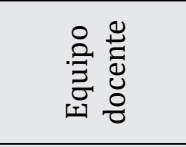 & \multirow{3}{*}{$\begin{array}{l}\text { Búsqueda de bibliografía, } \\
\text { lecturas y elección de } \\
\text { gestor de referencias } \\
\text { adecuado. } \\
\text { Identificación, y relación } \\
\text { de ideas y característi- } \\
\text { cas para fundamentar el } \\
\text { tema. } \\
\text { Reflexión sobre antece- } \\
\text { dentes, identificación } \\
\text { de lagunas, incorpora- } \\
\text { ción de información } \\
\text { adicional y solución de } \\
\text { incoherencias. }\end{array}$} & \multirow{3}{*}{$\begin{array}{l}\text { Definición de estructura } \\
\text { TFG. } \\
\text { Justificación de la relevancia } \\
\text { del tema. } \\
\text { Presentación de referencias } \\
\text { bibliográficas de fuentes } \\
\text { citadas. } \\
\text { Identificación de } \\
\text { descriptores. }\end{array}$} \\
\hline & & & $\begin{array}{l}\text { Exposición de la propuesta con- } \\
\text { creta por trabajar, objetivos y expe- } \\
\text { riencias analizadas. }\end{array}$ & & & \\
\hline & & & $\begin{array}{l}\text { Puesta en común de información } \\
\text { recogida por líneas temáticas en } \\
\text { pequeño grupo. }\end{array}$ & 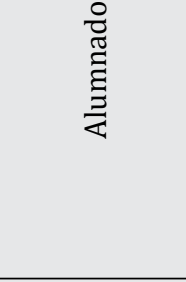 & & \\
\hline \multirow{3}{*}{ 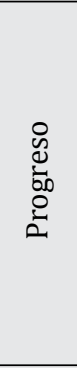 } & \multirow{3}{*}{10} & \multirow{3}{*}{ 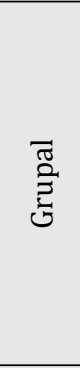 } & $\begin{array}{l}\text { Explicación de los puntos que deben } \\
\text { contener el diseño y la evaluación } \\
\text { de la propuesta de intervención }\end{array}$ & : & \multirow{3}{*}{$\begin{array}{l}\text { Orden de la producción } \\
\text { elaborada y conexión de } \\
\text { la fundamentación teó- } \\
\text { rica con planteamientos } \\
\text { desarrollados. } \\
\text { Elección de métodos tra- } \\
\text { bajo adecuados. }\end{array}$} & \multirow{3}{*}{$\begin{array}{l}\text { Elaboración de la introduc- } \\
\text { ción, estado cuestión y pro- } \\
\text { ceso metodológico. } \\
\text { Presentación de referencias } \\
\text { bibliográficas completas de } \\
\text { las fuentes citadas. } \\
\text { Planteamiento del } \\
\text { diseño de la propuesta } \\
\text { de intervención. } \\
\end{array}$} \\
\hline & & & $\begin{array}{l}\text { educativa (entrega documentación } \\
\text { elaborada para tal efecto). }\end{array}$ & 되 융 & & \\
\hline & & & $\begin{array}{l}\text { Puesta en común de información } \\
\text { sobre diseño de las propuestas por } \\
\text { líneas temáticas en pequeño grupo. }\end{array}$ & $\begin{array}{l}\frac{O}{0} \\
\stackrel{\pi}{\Xi} \\
\stackrel{\Xi}{\Xi} \\
\end{array}$ & & \\
\hline \multirow{4}{*}{$\begin{array}{l}0 \\
0 \\
\dot{\omega} \\
0 \\
0 \\
D\end{array}$} & \multirow{4}{*}{16} & \multirow{4}{*}{ 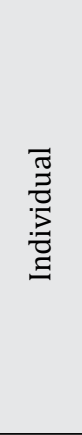 } & $\begin{array}{l}\text { Planteamiento de las dificultades } \\
\text { relacionadas con la elaboración de } \\
\text { la propuesta. }\end{array}$ & $\begin{array}{l}\frac{0}{\pi} \\
\stackrel{\pi}{\Xi} \\
\Xi\end{array}$ & \multirow{4}{*}{$\begin{array}{l}\text { Integración de la infor- } \\
\text { mación en discurso } \\
\text { coherente. } \\
\text { Establecimiento de rela- } \\
\text { ciones con conocimien- } \\
\text { tos previos, experiencias } \\
\text { análogas y principales } \\
\text { hallazgos del TFG. } \\
\text { Cuestionarse su trabajo y } \\
\text { las posibles extensiones } \\
\text { de este. }\end{array}$} & \multirow{4}{*}{$\begin{array}{l}\text { Redacción ordenada y con- } \\
\text { cisa de la propuesta (diseño } \\
\text { o evaluación). } \\
\text { Elaboración de } \\
\text { conclusiones, limitaciones } \\
\text { y prospectiva. } \\
\text { Concreción del resumen. } \\
\text { Presentación de referencias } \\
\text { bibliográficas y revisión del } \\
\text { estilo de citación. }\end{array}$} \\
\hline & & & & 完 & & \\
\hline & & & $\begin{array}{l}\text { Orientaciones sobre la elaboración } \\
\text { de conclusiones, limitaciones y } \\
\text { prospectiva. }\end{array}$ & $\begin{array}{l}\stackrel{ \pm}{\Xi} \\
\stackrel{\Xi}{0} \\
0 \\
0\end{array}$ & & \\
\hline & & & Resolución de dudas. & 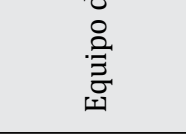 & & \\
\hline
\end{tabular}




\begin{tabular}{|c|c|c|c|c|c|c|}
\hline Fase & Semana & Tipo & Tema por tratar & Responsable & $\begin{array}{c}\text { Trabajo para el } \\
\text { estudiante }\end{array}$ & Evidencia escrita \\
\hline$\underset{\Xi}{\overparen{1}}$ & 18 & $\frac{\pi}{2}$ & $\begin{array}{l}\text { Cambios para introducir en el } \\
\text { informe final. }\end{array}$ & 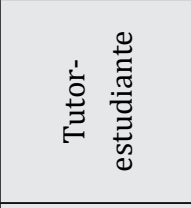 & $\begin{array}{l}\text { Revisión de la coheren- } \\
\text { cia entre las partes del } \\
\text { trabajo. }\end{array}$ & $\begin{array}{l}\text { Realización de las últimas } \\
\text { correcciones y mejoras del } \\
\text { TFG. } \\
\text { Entrega del TFG final al } \\
\text { tutor. }\end{array}$ \\
\hline$\underset{\Xi}{\overparen{\Xi}}$ & 20 & $\frac{\pi}{2}$ & $\begin{array}{l}\text { Cuestiones relacionadas con la } \\
\text { defensa del TFG. }\end{array}$ & 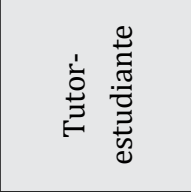 & $\begin{array}{l}\text { Exposición, de forma } \\
\text { ordenada y ajustada al } \\
\text { tiempo, de las aporta- } \\
\text { ciones realizadas por el } \\
\text { TFG. }\end{array}$ & $\begin{array}{l}\text { Elaboración de la presen- } \\
\text { tación y del ensayo de esta. }\end{array}$ \\
\hline
\end{tabular}

Fuente: elaboración propia.

\section{RESULTADOS}

Los TFG realizados han posibilitado un acercamiento al patrimonio histórico español mediante propuestas de ocio cultural; la socialización y la transmisión de tradiciones y juegos populares mediante los recreos activos; el fomento de la actividad física desde las primeras edades en el entorno natural, a través de los paseos saludables, y que la lectura se convierta en hábito para disfrutar en compañía.

Todas las iniciativas han ido más allá de un simple proyecto teórico. La motivación en la que se han visto inmersos los futuros maestros durante el desarrollo de sus TFG les ha llevado a un acercamiento experiencial en el ámbito profesional, al ofrecerles iniciativas en las esferas social y educativa.

En algunos casos, como en el del ocio cultural, se persigue favorecer el conocimiento y la valoración del patrimonio histórico-artístico realizando itinerarios por los lugares más emblemáticos de las ciudades de Logroño y Pamplona, y del pueblo riojano de Briones. Este proyecto plantea como principales objetivos: reconocer señas de identidad cultural, observar de forma activa el entorno, favorecer la relación familiar e incrementar el interés por participar en actividades sociales a través del desarrollo de una serie de rutas, como el Camino de Santiago a su paso por Logroño, la ruta interior por el Museo de La Rioja y exterior por las calles y las plazas de su capital, el paseo por los sitios más representativos de Pamplona y la visita al Museo Dinastía Vivanco, de Briones. Las rutas están diseñadas para hacerse en familia, con el acompañamiento de una educadora, quien presenta, de forma atractiva y pedagógica, el patrimonio histórico-artístico y fomenta la observación activa de los participantes.
Los recreos activos surgen como necesidad de recuperar juegos tradicionales, canciones populares y juegos motores y acercarlos a la población infantil y juvenil, a través de la implicación de la familia y, especialmente, de la figura de los abuelos. Esta propuesta plantea la realización de actividades que favorezcan el conocimiento, el respeto y la conservación de nuestra historia y nuestras costumbres, así como la promoción de relaciones sociales saludables. Como objetivos prioritarios, se plantean: acercar la cultura tradicional a través del juego, promover la participación de la comunidad educativa, impulsar el desarrollo de valores y promocionar relaciones sociales saludables. Apoyándose en el tiempo destinado al descanso de la jornada escolar, el recreo, se trata de transmitir y recuperar juegos tradicionales en la escuela y, asimismo, de promocionar relaciones sociales saludables a través de juegos motores, mediante la participación de la comunidad educativa.

La propuesta paseos saludables en familia pretende conciliar el tiempo de ocio familiar de padres e hijos mediante la actividad física durante la etapa de infantil implicando a los progenitores. Esta iniciativa se encuentra en estrecha consonancia con los Paseos Saludables de la Consejería de Salud y Servicios Sociales de la Comunidad Autónoma de La Rioja, con la particularidad de que en los paseos en familia prima la necesidad de intervenir en el tiempo libre de dicha institución. Teniendo en cuenta esas premisas, la iniciativa se propone: establecer una iniciativa social apoyada y sustentada en el diseño y la estructura de los "Paseos Saludables", fomentar el disfrute por la actividad física potenciando hábitos de vida saludables y reducir el sedentarismo o la inactividad física en los niños de entre 3-6 años. Este proyecto se lleva a cabo en la localidad riojana de Navarrete, y se compone de tres recorridos en familia ("Entre viñedos", 
"Camino de Santiago" y "Embalse de Valbornedo"), con diferentes rutas y actividades de carácter educativo y de salud para motivar a los más pequeños.

Por último, la iniciativa lectura en familia, bajo el título Leemos juntos, nos sentimos más seguros, se plantea como respuesta a la necesidad, latente en nuestra sociedad actual, de una formación en conocimientos básicos en primeros auxilios durante esta etapa, contemplada en nuestra actual Ley Orgánica para la Mejora de la Calidad Educativa (LOMCE, 2013). Se utiliza la lectura de relatos en el entorno familiar con la pretensión de: incrementar el tiempo de ocio saludable en familia, fomentar de forma indirecta el gusto por la lectura, dotar a los alumnos de estrategias que les permitan resolver situaciones en la cuales se demanden primeros auxilios y proporcionar una mayor empatía, mayor autonomía y mayor seguridad en sí mismos.

\section{CONCLUSIONES}

Estas iniciativas surgen de la necesidad de buscar nuevas fórmulas de tutoría y de formación teórico-práctica que orienten al profesorado y al alumnado universitario en el proceso de elaboración de los TFG en educación. La propuesta de tutoría que se plantea requiere: una buena planificación de los seminarios-talleres que ayuden al alumnado a regular el proceso de elaboración del TFG; el establecimiento de unas directrices, por parte de los tutores, que aporten claridad; la creación de un ambiente de confianza, seguridad y respeto mutuo, en el que se consideren todas las opiniones y los estudiantes tengan el feedback adecuado de los tutores, y una buena coordinación y organización del equipo docente, donde la formación de equipos de trabajo multidisciplinares y el trabajo en red son de los elementos clave de la propuesta, que ha permitido promover iniciativas colectivas y optimizar el trabajo de los tutores. De todo ello depende la calidad del TFG.

Si bien al principio del proceso el alumnado se muestra expectante, dada la dinámica de trabajo, la evaluación realizada hasta el momento indica que el alumnado valora de manera positiva los talleres-seminarios como recurso educativo para intercambiar ideas, formas de pensar y actuar (Soria, 2013) y contrastar experiencias, así como valorar la importancia del trabajo en equipo, desarrollar un compromiso de responsabilidad frente a sus compañeros, activar procesos reflexivos críticos sobre la propia práctica y adquirir destrezas para trabajar de manera interdisciplinar desde la planificación al diseño, la intervención y la evaluación de las diferentes propuestas de ocio saludable familiar (Sáenz de Jubera, Alonso, Sanz, Ponce de León \& Valdemoros, 2015). De todas formas, se ha observado que presentan dificultades para gestionar el tiempo de trabajo y para cumplir con el cronograma que se les propone. Esto llevará a presentar, de manera razonada y desde la primera sesión, las fases previstas para la realización del TFG y su cronograma asociado, así como a hacer hincapié en la necesidad de su cumplimiento.

Asimismo, la acción tutorial en el proceso de elaboración de los TFG ha posibilitado, por un lado, la consecución de importantes resultados de aprendizaje asociados a esta asignatura y, por otro, el acercamiento del alumnado a experiencias profesionales, de modo que el TFG se ha convertido en el escenario idóneo en el que desarrollar su futuro laboral inmediato.

Por la acogida y las opiniones vertidas por los docentes de los centros educativos donde se han implementado las propuestas, así como de los beneficiarios directos, se concluye que con estas acciones se han obtenido interesantes ventajas educativas, sociales y culturales, por lo que interesa hacerlas transferibles a otros contextos, que se resumen en los siguientes puntos:

- Son un recurso educativo y lúdico.

- Su coste económico es bajo.

- Utilizan una metodología activa y participativa.

- Desarrollan espacios para el aprendizaje y la acción didáctica, en consonancia con Bygren et al. (2009) y Elchardus (2009).

- Favorecen la expresión de la creatividad, como preconiza Cuenca (2000).

- Facilitan el intercambio intergeneracional, como así lo defienden Álvarez y García (2013), o el intercultural, como lo hacen Katz-Gerro, Raz y Yaish (2009).

- Son diseñadas para implementarse desde espacios formales a otros más informales. 


\section{REFERENCIAS}

Agate, J. R., Zabriskie, R. B., Agate, S. T, \& Poff, R. (2009). Family Leisure Satisfaction and Satisfaction with Family Life. Journal of Leisure Research, 41 (2), 205223. Recuperado de: http://js.sagamorepub.com/jlr/ article/view/418.

Agate, S. T., Zabriskie, R. B. \& Eggett, D. (2007). Praying, playing, and successful families: An examination of family religiosity, family leisure, and family functioning. Marriage and Family Review, 42 (2), 5175. DOI: 0.1300/J002v42n02_04.

Álvarez, C. \& García, M. (2013). Escuela, museo, familia, lectura y valores: Proyecto de educación en valores para la Red de Museos Etnográficos de Asturias. Íber. Didáctica de las Ciencias Sociales, Geografía e Historia, 73 (1), 73-80. Recuperado de: http://goo.gl/YvAj0b.

Álvarez, J. \& Rodríguez, C. (2008). El valor de la institución familiar en los jóvenes universitarios de la Universidad de Granada. Bordón. Revista de pedagogía, 60 (1), 7-21. Recuperado de: http://goo.gl/F6mKLr.

Álvarez, M., Fernández-Villarán, A. \& Mendoza, L. (2014). Ocio como ámbito de socialización juvenil. En C. Ortega \& F. Bayón (Coords.). El papel del ocio en la construcción social del joven (pp. 97-123). Bilbao: Universidad de Deusto.

Bygren, L. O., Weissglas, G., Wikström, B. M., Konlaan, B. B., Grjibovski, A., Karlsson, A. B., Andersson, S. O. \& Sjöström, M. (2009). Cultural participation and health: a randomized controlled trial among medical care staff. Psychosomatic Medicine, 71 (4), 469-473. doi: 10.1097/PSY.0b013e31819e47d4.

Caride, J. A. (1998). Educación del ocio y del tiempo libre. En M. Beas \& J. García (Coords.). Atención a los espacios y tiempos escolares (pp. 17-31). Granada: Grupo Editorial Universitario.

Caride, J. A. (2012). Lo que el tiempo educa: el ocio como construcción pedagógica y social. Arbor, 188, 301-313. DOI: 10.3989/arbor.2012.754n2001.

Carrera, E. (2009). Asociacionismo en el tiempo libre. En J. C. Otero (Ed.), La pedagogía del ocio: Nuevos desafíos (pp. 9-23). Lugo: Axac.

Clerton de Oliveira, J., Francileudo, F. A. \& Ibiapina, L. (2014). El tiempo de ocio para el estilo de vida contemporáneo: significados a lo largo de la vida. En C. Ortega \& F. Bayón (Coords.). El papel del ocio en la construcción social del joven (pp. 61-77). Bilbao: Publicaciones de la Universidad de Deusto.

Cuenca, M. (1997). La intervención educativa en ocio y tiempo libre. En AAVV: Nuevos espacios de la educación social (pp. 253-286). Bilbao: Universidad de Deusto.
Cuenca, M. (2000). Ocio humanista. Dimensiones y manifestaciones actuales del ocio. Bilbao: Publicaciones de la Universidad de Deusto.

Cuenca, M. (2009). Ocio humanista. Dimensiones y manifestaciones actuales del ocio. Bilbao: Universidad de Deusto.

Cuenca, M. (2013). Ocio valioso en tiempos de crisis. En S. Torío, O. García-Pérez, J. V. Peña \& C. M. Fernández (Coords.). La crisis social y el estado del bienestar: las respuestas de la pedagogía social (pp. 5-20). Oviedo: Universidad de Oviedo.

Cuenca, M. (2014). Ocio valioso. Bilbao: Universidad de Oviedo.

Cuenca, M. \& Goytia, A. (2012). Ocio experiencial: antecedentes y características. Arbor, 188, 265-281. DOI: 10.3989/arbor.2012.754n2001.

Elchardus, M. (2009). Self-control as social control: the emergence of symbolic society. Poetics, 37 (2), 146161. DOI: 10.1016/j.poetic.2009.01.001.

Flores, C., Martínez-Berbel, J. A., Martínez, M. I., Pascual, N. \& Sanz, E. (2013). Manual de orientación para la tutorización y evaluación del Trabajo Fin de Grado. Logroño: Universidad de La Rioja. Recuperado de http://dialnet.unirioja.es/servlet/ libro? codigo $=528090$.

Katz-Gerro, T., Raz, S. \& Yaish, M. (2009). How do class, status, ethnicity, and religiosity shape cultural omnivorousness in Israel? Journal of Cultural Economics, 33 (1), 1-17. Recuperado de: http://goo. gl/Rrv3bJ.

Ley orgánica para la mejora de la calidad educativa (LOMCE) (Ley Orgánica 8/2013, 9 de diciembre). Boletín Oficial del Estado, 295, 2013.

Mactavish, J. \& Schleien, S. (1998). Playing together growing together: Parents' perspectives on the benefits of family recreation in families that include children with a developmental disability. Therapeutic Recreation Journal, 32 (3), 207-230. Recuperado de: http://goo.gl/bNHYrZ.

Otero, J. A. (2009). La pedagogía del ocio: nuevos desafíos. Lugo: Axac.

Ponce de León, A., Sanz, E. \& Valdemoros, M. A. (2013). El trabajo fin de grado, marco para el desarrollo del emprendimiento entre los jóvenes. En S. Torío, 0. García, J. V. Peña y C. M. Fernández (Coords.). La crisis social y el estado del bienestar: las respuestas de la Pedagogía Social (pp. 324-328). Oviedo: Universidad de Oviedo.

Quintana, J. M. (1991). El tiempo libre como ámbito humano y cultural. En J. M. Quintana, Iniciativas 
sociales en educación informal (pp. 402-415). Madrid: Rialp.

Real Decreto 861/2010, de 2 de julio, por el que se modifica el Real Decreto 1393/2007, de 29 de octubre, por el que se establece la ordenación de las enseñanzas universitarias oficiales.

Rekalde, M. I. (2011). ¿Cómo afrontar el trabajo fin de grado?: Un problema o una oportunidad para culminar con el desarrollo de las competencias. Revista complutense de educación, 22 (2), 179-193.

Sáenz de Jubera, M., Alonso, R. A., Sanz, E., Ponce de León, A. \& Valdemoros, M. A. (2015). Ocio saludable en familia. Una apuesta para los Trabajos Fin de Grado en Educación. Actas de las I Jornadas de Innovación Docente Campus Iberus y IX Jornadas de Innovación Docente e Investigación Educativa UZ. Zaragoza: Universidad de Zaragoza. Recuperado de: https://goo. $\mathrm{gl} / \mathrm{kK} 6 \mathrm{oGn}$.
Soria, V. (2013). Conclusiones y recomendaciones sobre las actividades formativas del TFG. Actas de la Jornada sobre el Trabajo de Fin de Grado. Zaragoza: Universidad de Zaragoza. Recuperado de: http://goo.gl/Et3HrZ.

Valdemoros, M. A., Ponce de León A., Sanz, E. \& Caride, J. A. (2014). La influencia de la familia en el ocio físico-deportivo juvenil: nuevas perspectivas para la reflexión y la acción. Arbor, (770)190-192. DOI: http://dx.doi.org/10.3989/arbor.2014.770n6013.

World Leisure and Recreation Association (1994). International Charter for Leisure Education. European Leisure and Recreation Association (ELRA), Summer, 13-16.

Zabriskie, R. B. \& McCormick, B. P. (2001). The Influences of Family Leisure Patterns on Perceptions of Family Functioning. Family Relations, 50, 281-289. DOI: 10.1111/j.1741-3729.2001.00281.x.

Zabriskie, R. B. \& McCormick, B. P. (2003). Parent and child perspectives of family leisure involvement and satisfaction with family life. Journal of Leisure Research, 35 (2), 163-189. Recuperado de: http:// js.sagamorepub.com/jlr/article/view/604. 